\title{
Iron oxychloride as an efficient catalyst for selective hydroxylation of benzene to phenol
}

EIMetwally, Ahmed E.; Eshaq, Ghada; Yehia, Fatma Z.; Al-Sabagh, Ahmed M.; Kegnæs, Søren

Published in:

ACS Catalysis

Link to article, DOI:

10.1021/acscatal.8b03590

Publication date:

2018

Document Version

Peer reviewed version

Link back to DTU Orbit

Citation (APA):

ElMetwally, A. E., Eshaq, G., Yehia, F. Z., Al-Sabagh, A. M., \& Kegnæs, S. (2018). Iron oxychloride as an efficient catalyst for selective hydroxylation of benzene to phenol. ACS Catalysis, 8, 10668-10675.

https://doi.org/10.1021/acscatal.8b03590

\section{General rights}

Copyright and moral rights for the publications made accessible in the public portal are retained by the authors and/or other copyright owners and it is a condition of accessing publications that users recognise and abide by the legal requirements associated with these rights.

- Users may download and print one copy of any publication from the public portal for the purpose of private study or research.

- You may not further distribute the material or use it for any profit-making activity or commercial gain

- You may freely distribute the URL identifying the publication in the public portal 


\section{Article}

Subscriber access provided by DTU Library

\section{Iron oxychloride as an efficient catalyst for selective hydroxylation of benzene to phenol}

Ahmed E. ElMetwally, Ghada Eshaq, Fatma Z. Yehia, Ahmed M. Al-Sabagh, and Søren Kegnæs ACS Catal., Just Accepted Manuscript • DOI: 10.1021/acscatal.8b03590 • Publication Date (Web): 09 Oct 2018

Downloaded from http://pubs.acs.org on October 15, 2018

\section{Just Accepted}

"Just Accepted" manuscripts have been peer-reviewed and accepted for publication. They are posted online prior to technical editing, formatting for publication and author proofing. The American Chemical Society provides "Just Accepted" as a service to the research community to expedite the dissemination of scientific material as soon as possible after acceptance. "Just Accepted" manuscripts appear in full in PDF format accompanied by an HTML abstract. "Just Accepted" manuscripts have been fully peer reviewed, but should not be considered the official version of record. They are citable by the Digital Object Identifier (DOI®). "Just Accepted" is an optional service offered to authors. Therefore, the "Just Accepted" Web site may not include all articles that will be published in the journal. After a manuscript is technically edited and formatted, it will be removed from the "Just Accepted" Web site and published as an ASAP article. Note that technical editing may introduce minor changes to the manuscript text and/or graphics which could affect content, and all legal disclaimers and ethical guidelines that apply to the journal pertain. ACS cannot be held responsible for errors or consequences arising from the use of information contained in these "Just Accepted" manuscripts. 


\title{
Iron oxychloride as an efficient catalyst for selective hydroxylation of benzene to phenol
}

\author{
Ahmed E. EIMetwally*, a, Ghada Eshaq a, Fatma Z. Yehia a , Ahmed M. Al-Sabagh b, Søren \\ Kegnæs ${ }^{c}$ \\ ${ }^{a}$ Petrochemicals department, Egyptian Petroleum Research Institute, Nasr City, Cairo 11727, Egypt \\ b Petroleum applications department, Egyptian Petroleum Research Institute, Nasr City, Cairo 11727, Egypt \\ c DTU Chemistry, Technical University of Denmark, Kemitorvet 207, DK-2800 Kgs. Lyngby, Denmark
}

\begin{abstract}
Selective hydroxylation of benzene is a felicitous strategy for the production of phenol that is deemed an alternative route for conventional processes. Thus, the development of a durable and highly efficient catalyst for the selective hydroxylation of benzene should be the key topic. In this work, $\mathrm{FeOCl}$ was prepared by chemical vapor transition method and characterized using various techniques including XRD, TEM, Raman spectroscopy, $\mathrm{N}_{2}$ adsorption-desorption, DLS and TGA. The prepared $\mathrm{FeOCl}$ was applied as heterogeneous catalyst in benzene hydroxylation and the reaction conditions were optimized. The acquired data manifested that $\mathrm{FeOCl}$ has shown superiority over the other reported catalysts utilized in benzene hydroxylation. The superiority of $\mathrm{FeOCl}$ is attributed to the facile self-redox potential of $\mathrm{FeOCl}$ and its remarkable ability for the production of an overwhelming amount of hydroxyl radicals in a short period of time. The catalyst recovery and reusing test showed that $\mathrm{FeOCl}$ is able to endure the harsh conditions of benzene hydroxylation for four runs. The mechanism of benzene hydroxylation using $\mathrm{FeOCl}$ as a catalyst in the presence of hydrogen peroxide as an oxidant was also illustrated.
\end{abstract}

Keywords: benzene; phenol; hydroxylation; catalysis; oxychloride

*corresponding author: Tel: +201115025588, Fax: +20222747433 
E-mail address: ahmed_ezzatt@msn.com

\section{INTRODUCTION}

Phenol is deemed as one of the most indispensable organic compound that is used extensively in various chemical and petrochemical industries. Phenol is also used as an intermediate in the production of phenol derivatives and phenolic resins. Cumene process is commonly used for the production of nearly $90 \%$ of phenol produced throughout the globe, where propylene and benzene are used as starting materials with acetone as a side product. $^{1-2}$ Cumene process comprises three consecutive stages and suffers from various drawbacks including excessive energy consumption and the generation of hazardous intermediate such as cumene hydroperoxide. In spite of the economic importance for the production of acetone, the demand for phenol is still rising as the global demand for phenol is currently being driven by an increasing demand of its various derivatives. ${ }^{1,3}$ Consequently, the production of phenol from the direct oxidation of benzene has attracted much attention to overcome the drawbacks encountered with cumene process. ${ }^{4-6}$

Recently, great efforts have been made to develop the catalytic system of the direct oxidation of benzene to phenol especially when the hydrogen peroxide is used as an oxidant. ${ }^{7}$ During this tenure, several catalysts including palladium membrane, modified titanium silicalite, titanium-mesoporous molecular sieves, vanadium-substituted polyoxometalates, activated carbon, cerium promoted $\mathrm{V}-\mathrm{g}-\mathrm{C}_{3} \mathrm{~N}_{4}$ and vanadium-containing mesoporous carbon have been explored. ${ }^{8-22}$ Among these explored catalysts, iron-based catalysts have shown supremacy over the other tested catalysts in different aspects including the cost, abundance, environmental friendliness and low toxicity. 
Previous studies reported that iron-based catalysts were highly efficient and selective when used as catalysts in different oxidative processes and particularly the challenging processes. ${ }^{23-25}$ Wang et al. ${ }^{26}$ reported that a high phenol yield from benzene hydroxylation can be achieved over two Fe-based metal-organic frameworks catalysts using hydrogen peroxide as an oxidant. Jourshabani et al. ${ }^{27}$ studied the effect of using Fe-supported cage like mesoporous silica in benzene hydroxylation to phenol using $\mathrm{H}_{2} \mathrm{O}_{2}$ as an oxidant. Baykan and Oztas, ${ }^{28}$ reported the synthesis of phenol via the direct hydroxylation of benzene over iron phosphate. Carneiro and Silva, ${ }^{29}$ studied the direct hydroxylation of benzene to phenol using hydrogen peroxide in the presence of Fe(III) Schiff base complex as a catalyst. Recently, iron ferrite was reported to be an efficient catalyst for the selective hydroxylation of benzene to phenol under mild conditions in the presence of hydrogen peroxide as an oxidant. ${ }^{30}$ Although the examined catalysts were efficient and selective in benzene hydroxylation, the phenol yield did not reach its climax as this process still demand more development. Thus, exploring a catalyst that is able to enhance the phenol yield remains a major goal. Such catalyst will be able to minimize the production of different byproducts while maintaining a high phenol yield.

Iron oxychloride $(\mathrm{FeOCl})$ is a ternary layered material that have distinguished layered framework and unrivaled chemical stability. The layers of this distinguishable layered framework are attached together by van der Waals interaction, which makes $\mathrm{FeOCl}$ able to host organic compounds. ${ }^{31}$ The charge transfers between the host organic compound and $\mathrm{FeOCl}$ may change iron oxidation state from +3 to $+2 .{ }^{32}$ Studies revealed that hosting an organic compound within $\mathrm{FeOCl}$ layered framework was responsible for the in situ reduction of one fourth of $\mathrm{Fe}^{3+}$ to $\mathrm{Fe}^{2+} .{ }^{32-}$

${ }^{33} \mathrm{FeOCl}$ has been employed as a catalyst in the degradation of some persistent organic compound 
and found to have fabulous activity, which is attributed to the extraordinary structural configuration of its atoms and also for its reducible electronic characteristics. ${ }^{34}$

We believe that this is the first article that uses $\mathrm{FeOCl}$ as a heterogeneous catalyst in benzene hydroxylation to phenol. Herein, $\mathrm{FeOCl}$ was prepared using chemical vapor transition method and characterized using XRD, TEM, Raman spectroscopy, $\mathrm{N}_{2}$ - sorption, DLS and TGA analysis. $\mathrm{FeOCl}$ was examined in benzene hydroxylation and the reaction conditions were optimized to fulfil the maximum phenol yield. Finally, the reaction mechanism of benzene hydroxylation using FeOCl was illustrated.

\section{EXPERIMENTAL}

\section{Materials}

Anhydrous iron (III) chloride and ethylene glycol were supplied from Sigma-Aldrich. Benzene and ammonia solution (30\%) were supplied from Merck. Hydrogen Peroxide (30\%) was supplied from PanReac AppliChem. Acetic Acid (70-80\% w/w) was supplied from Fisher Scientific.

\section{Preparation of FeOCl}

First, $\alpha-\mathrm{Fe}_{2} \mathrm{O}_{3}$ was prepared by dissolving certain amount of anhydrous ferric chloride in aqueous solution containing $80 \%$ ethylene glycol. Ferric ions were precipitated by adding $3 \mathrm{M}$ ammonia solution under stirring at $50^{\circ} \mathrm{C}$ and kept at this temperature for $30 \mathrm{~min}$. The formed slurry was aged for $12 \mathrm{~h}$ and then the resultant precipitate was separated by centrifugation, washed by deionized water and dried at $100^{\circ} \mathrm{C}$ overnight. Second, $\mathrm{FeOCl}$ was prepared by chemical vapor transition method from the prepared $\mathrm{Fe}_{2} \mathrm{O}_{3}$ according to the procedure reported in. ${ }^{34}$ In particular, the prepared $\alpha-\mathrm{Fe}_{2} \mathrm{O}_{3}$ was mixed with anhydrous ferric chloride in ratio of $1: 1.3$. The resultant mixture was sealed in an evacuated glass vessel and kept in a muffle furnace for $48 \mathrm{~h}$ at $380^{\circ} \mathrm{C}$. 
The obtained sample was washed successively with acetone to get rid of the unreacted ferric chloride and eventually dried at $100{ }^{\circ} \mathrm{C}$ for $6 \mathrm{~h}$.

\section{Characterization}

A JEOL JEM-2 100 operating at $200 \mathrm{kV}$ was used to obtain the TEM images of the sample.

The hydrodynamic diameter was characterized using dynamic light scattering (DLS) analysis on a Malvern Zetasizer Nano (Malvern Instruments Ltd., Worcestershire, UK). XRD analysis was performed to identify and confirm the phase of the prepared sample using a Philips powder diffractometer using $\mathrm{Cu} \mathrm{K \alpha}$ radiation. The analysis was carried out at a scanning speed of $2^{\circ} \theta / \mathrm{min}$ and $2 \theta$ step of $0.02^{\circ}$. The Raman spectra of the prepared sample was obtained using a Bruker Optics Raman spectrometer operating at room temperature with an exciting lines of $532 \mathrm{~nm}$. TGA analysis was conducted at rate of $10^{\circ} \mathrm{C} \mathrm{min}^{-1}$ under nitrogen atmosphere on a SDT Q600, TA Instruments. A NOVA 3200 system instrument was used to obtain the surface texture parameters by nitrogen sorption at $-195.85^{\circ} \mathrm{C}$.

\section{Catalytic activity}

The reaction was carried out in a $25 \mathrm{~mL}$ two necked flask equipped with a condenser. The reaction temperature was adjusted by immersing the flask in a thermally controlled oil bath. In each run, benzene $(0.9 \mathrm{~mL})$ and $70 \%$ acetic acid $(1 \mathrm{~mL})$ were fed into the two necked flask followed by the addition of a particular amount of FeOCl. Thereafter, certain amount of 30\% hydrogen peroxide was introduced to the reaction medium. The catalytic activity was evaluated at different reaction temperature in the range of $30-80^{\circ} \mathrm{C}$ and at different reaction time in the range of 0.5-6 h. When the reaction was complete, the catalyst was separated by centrifugation and the liquid product was collected to be analyzed. An Agilent 7890 GC equipped with HP-5 capillary 
column (5\%-Phenyl)-methylpolysiloxane $(30 \mathrm{~m}, 0.25 \mu \mathrm{m}$ and $0.32 \mathrm{~mm})$ and flame ionization detector. The phenol selectivity was obtained as the molar ratio of the produced phenol to the converted benzene while the phenol yield was obtained as the molar ratio of the produced phenol to the initial benzene. $p$-benzoquinone selectivity was obtained as the molar ratio of the produced p-benzoquinone to the converted benzene. ${ }^{35}$

\section{Results and Discussion}

\section{Catalyst characterization}

The TEM micrograph of the prepared sample reveals that catalyst has a stone-like morphology with a size in the range of $40-50 \mathrm{~nm}$ as displayed in Figure 1.

Dynamic light scattering technique was conducted to screen the size distribution of the prepared $\mathrm{FeOCl}$. The obtained data reveal that the diameter range of $\mathrm{FeOCl}$ is $50 \mathrm{~nm}$ as displayed in Figure 1.

The phase of the prepared $\mathrm{FeOCl}$ sample was identified and confirmed using the obtained $\mathrm{XRD}$ pattern presented in Figure 1. The phase of the prepared $\mathrm{FeOCl}$ is confirmed by matching the obtained pattern with the FeOCl reference code (01-072-0619). The obtained data manifest that the prepared $\mathrm{FeOCl}$ has an orthorhombic structure with a crystallite size of $50 \mathrm{~nm}$ that was calculated using Scherrer equation, which is in consistence with the data observed in the TEM micrograph and DLS analysis.

The Raman spectrum of the prepared $\mathrm{FeOCl}$ (Figure 1) exhibits band at $216 \mathrm{~cm}^{-1}$ that can be ascribed to $\mathrm{Fe}-\mathrm{Cl}$ stretching modes while the bands appear at $285 \mathrm{~cm}^{-1}$ and $400 \mathrm{~cm}^{-1}$ can be ascribed to $\mathrm{Fe}-\mathrm{O}$ stretching modes.

The acquired $\mathrm{N}_{2}$ sorption isotherm of the prepared $\mathrm{FeOCl}$ reveals that the sample can be categorized to Type II as shown in Figure 1. The isotherm of FeOCl exhibits an initial knee, which 
is attributed to the monolayer formation. It should be mentioned that the region observed for the onset of multilayer formation is usually recognized with initial concaved portion followed by a hoist that ascribed to multilayer formation. It is obvious that the $\mathrm{FeOCl}$ isotherm exhibits an $\mathrm{H} 3$ hysteresis that extends $\mathrm{P} / \mathrm{P}^{\circ}=0.15$. These characteristics can be ascribed to the existence of fragile narrow pores that imploded during the formation of monolayer leading to the formation of an open texture with a slit shape where a limited numbers of multilayer is formed. ${ }^{36-37}$ The value of specific surface area calculated using BET equation for $\mathrm{FeOCl}$ is $17.11 \mathrm{~m}^{2} \mathrm{~g}^{-1}$, and the total pore volume $(\mathrm{Vp})$ taken at $\mathrm{P} / \mathrm{Po}=0.95$, is $0.034, \mathrm{~mL} \mathrm{~g}^{-1}$. The $\mathrm{BJH}$ calculation method was used to obtain the pore size distribution curves as shown in Figure 1. The pore size distribution curve of iron oxychloride implies the presence of a sharp peak with a climax centered at $19 \AA$ and broad peak with a climax centered at $46 \AA$.

The thermal stability analysis was carried out prior to the catalytic testing to make sure the catalyst is able to endure the reaction temperature of benzene hydroxylation. It also provides a preliminary survey whether the reusability of the employed catalyst is possible or not. The TGA analysis of the prepared $\mathrm{FeOCl}$ (Figure 1) reveals that the catalyst endures the examined temperature range without any obvious transformational change. Thus, it can be inferred that the employed catalyst is thermally stable within the range of the tested reaction temperature of benzene hydroxylation. 

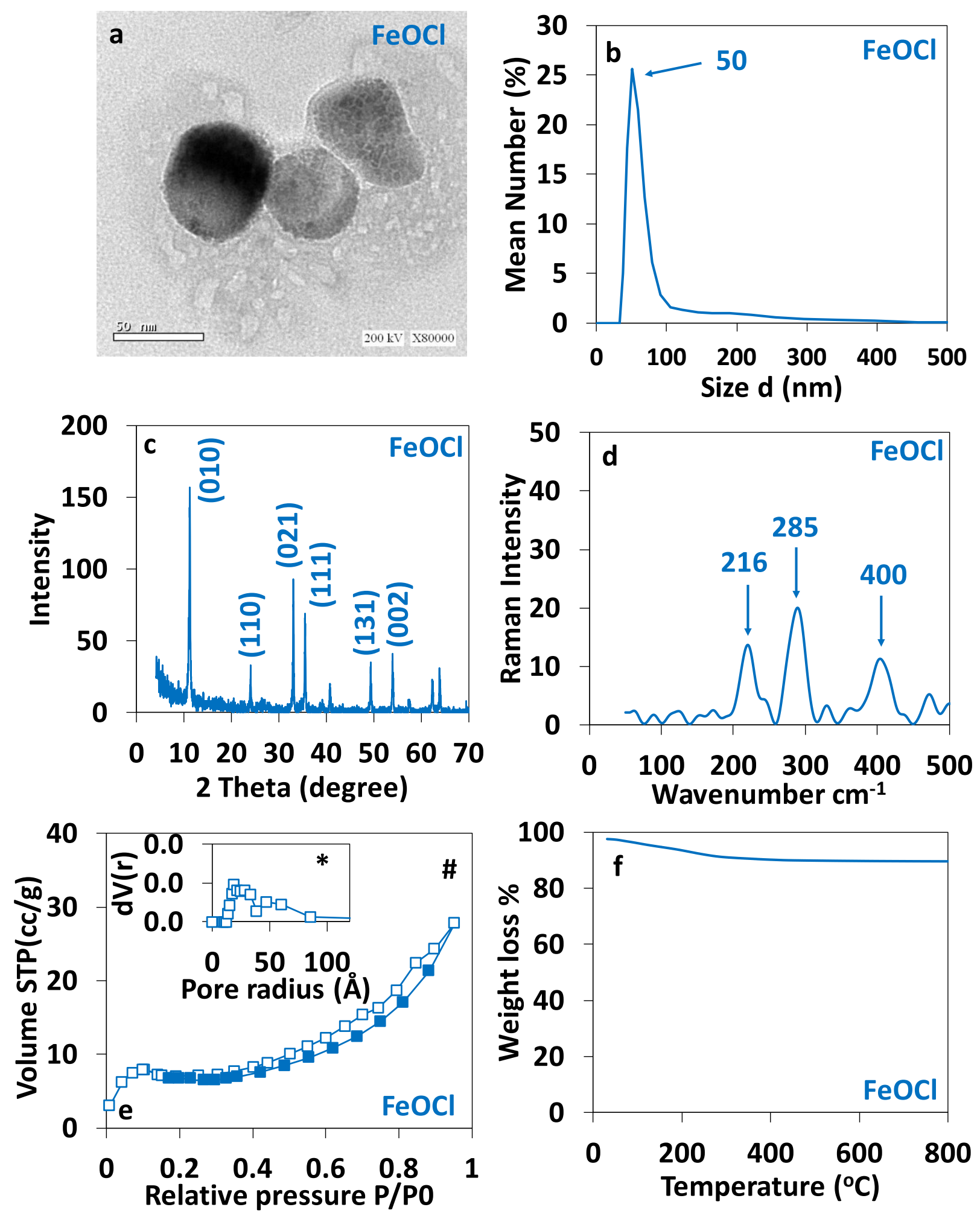

Figure 1. (a) TEM image of FeOCl. (b) DLS of FeOCl (c) XRD pattern of FeOCl. (d) Raman spectrum of FeOCl. (e\#) $\mathrm{N}_{2}$ adsorption-desorption isotherm of $\mathrm{FeOCl}\left(\mathrm{e}^{*}\right)$ pore size distribution curve of $\mathrm{FeOCl}$. (f) TGA curve of $\mathrm{FeOCl}$. 


\section{The catalytic activity of $\mathrm{FeOCl}$ in benzene hydroxylation Impact of reaction time}

The impact of reaction time is a very important parameter that gives us a wide view on the catalytic performance of the catalyst. When the phenol yield becomes steady or decreases this means that either the radical chain reaction is brought to an end or the generated phenol is further oxidized. The impact of reaction time on benzene hydroxylation was tested in the $0.5-6 \mathrm{~h}$ range as presented in Figure 2 and Table S1. The obtained data reveal that the phenol yield is directly proportional to the reaction time reaching a climax with a value of $43.5 \%$ after $4 \mathrm{~h}$ of benzene hydroxylation and beyond this time the phenol yield slightly decreases. Consequently, the use of elongated time of benzene hydroxylation has a negative impact on the phenol yield because the produced phenol undergoes further oxidation. Thus, it can be inferred that beyond $4 \mathrm{~h}$ of benzene hydroxylation a significant portion of the generated radical species tends to oxidize the produced phenol molecules instead of attacking benzene molecules. This explanation agrees well with the data obtained for phenol selectivity upon increasing the reaction time, which reveals a slight decrease in the phenol selectivity due to the production of $p$-Benzoquinone (Table S2 and Figure $\mathrm{S} 1)$ as a side product. $p$-Benzoquinone is a stable side product produced as result of hydroquinone oxidation. 


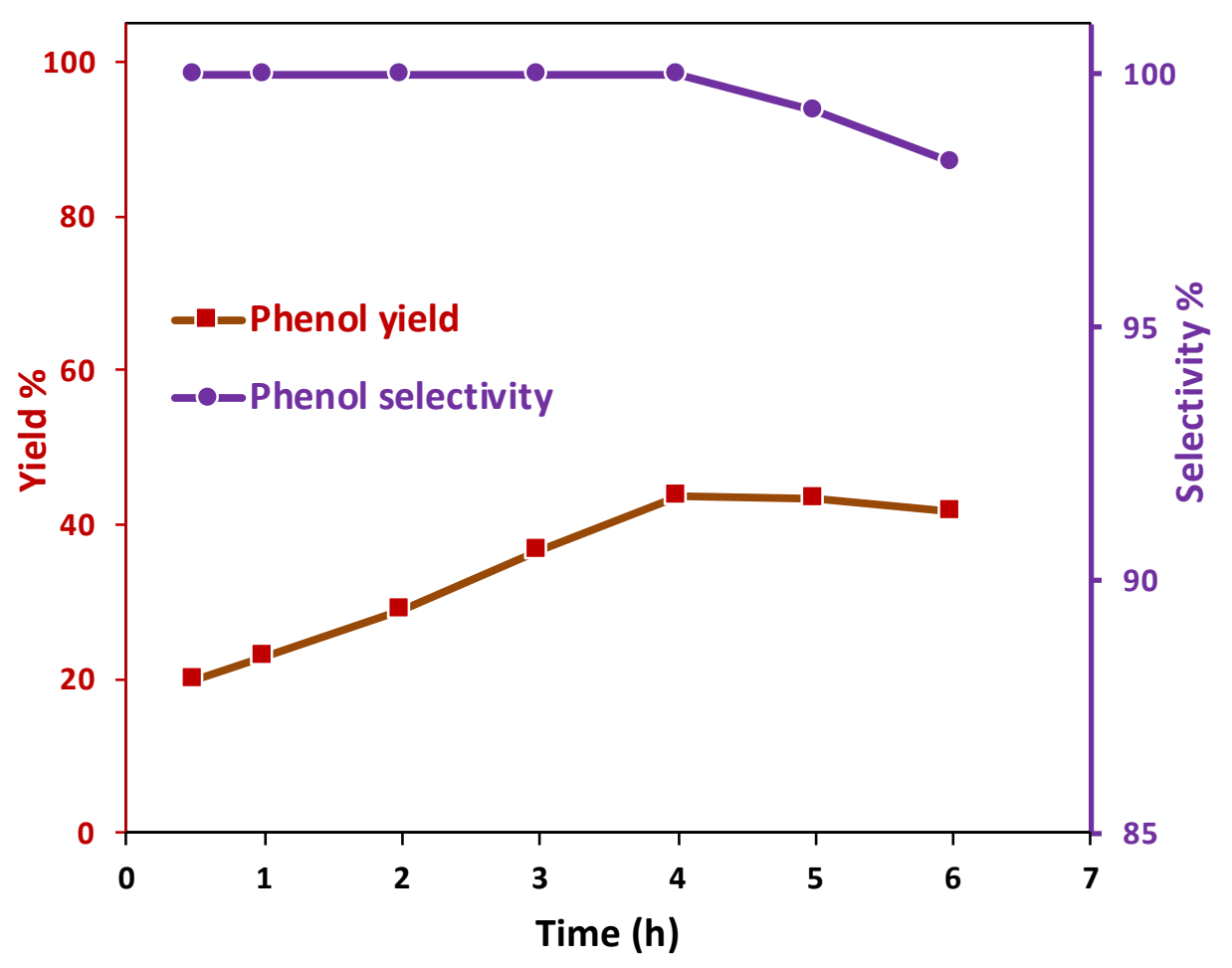

Figure 2. Impact of reaction time. Reaction conditions: Benzene $(0.9 \mathrm{~mL}), \mathrm{FeOCl}(0.1 \mathrm{~g})$, benzene: $\mathrm{H}_{2} \mathrm{O}_{2}(1: 1)$, acetic acid $(1 \mathrm{~mL})$ and $60^{\circ} \mathrm{C}$.

Compared to the previously reported data (Table S3), a maximum phenol yield of $43.5 \%$ in only $4 \mathrm{~h}$ of benzene hydroxylation highlights the high efficacy of FeOCl. This high efficacy can be attributed to the unique ability of $\mathrm{FeOCl}$ for the production of a high amount of hydroxyl radicals in a short period of time. This ability can be ascribed to the substantial structure of FeOCl where its polar surface and specifically the 020 plane is overstuffed with a huge number of unsaturated Fe atoms. ${ }^{38}$ Compared to water, the hydrogen peroxide molecules have more affinity to the polarized plan of iron oxychloride. ${ }^{34}$ These Fe atoms were uniformly arranged on this plan with a $\mathrm{O}-\mathrm{Fe}-\mathrm{Cl}$ configuration. 
Compared to $\alpha-\mathrm{FeOOH}, \mathrm{FeOCl}$ is able to increase the production of hydroxyl radicals from hydrogen peroxide with a yield 100 times higher than that produced in the presence of $\alpha-\mathrm{FeOOH}{ }^{34}$ Moreover, the $\mathrm{Fe}^{3+}$ ions of the iron oxychloride are susceptible to be reduced to $\mathrm{Fe}^{2+}$ ions upon their reaction or intercalation with different species, which makes clear that $\mathrm{FeOCl}$ is distinguished by facile self-redox properties. The $\mathrm{Fe}^{2+}$ ions are able to catalyze the production of hydroxyl radicals from hydrogen peroxide with a rate 1000-10000 times higher than that produced in the presence of $\mathrm{Fe}^{3+}$ ions. ${ }^{39} \mathrm{~A}$ partial reduction could occur as a result of the electron-charge transition from hydrogen peroxide to $\mathrm{FeOCl}$ upon the adsorption of hydrogen peroxide and organic molecules over the $\mathrm{FeOCl}$ surface.

$$
\begin{aligned}
& \mathrm{FeOCl}+\mathrm{H}_{2} \mathrm{O}_{2} \leftrightarrow \mathrm{Fe}_{1-n}{ }^{\mathrm{III}} \mathrm{Fe}_{n}{ }^{\mathrm{II}}+\mathrm{HOO}^{\bullet}+\mathrm{H}^{+} \\
& \equiv \mathrm{Fe}^{\mathrm{II}}+\mathrm{H}_{2} \mathrm{O}_{2} \leftrightarrow \equiv \mathrm{Fe}^{\mathrm{III}}+\mathrm{HO}^{\bullet}+\mathrm{OH}^{-}
\end{aligned}
$$

Thus, the efficacy of $\mathrm{FeOCl}$ in benzene hydroxylation can be attributed to unique features of $\mathrm{FeOCl}$ and the matchless configuration.

\section{Impact of catalyst dosage}

To find out the most suitable catalyst dosage on phenol yield, benzene hydroxylation reaction was carried out using different catalyst doses in the $0.05-0.25 \mathrm{~g}$ range as displayed in Figure 3. The results show that the phenol yield increases with increasing the catalyst dosage and it reaches a maximum value at a catalyst dosage of $0.1 \mathrm{~g}$ and beyond this amount the phenol yield decreases. It is clear that increasing the catalyst dosage has a positive impact on the phenol yield because of increasing the number of active sites that leads to a massive increase in the amount of hydroxyl radicals. However, the addition of an excessive amount of catalyst has a negative impact 
on the phenol yield because of the agglomeration of the catalyst particles that block the active sites. This blockage prevents the benzene molecules from reaching the active sites that are rich with highly active hydroxyl radicals and consequently the hydroxyl radicals tend to scavenge each other instead of attacking the benzene molecules.

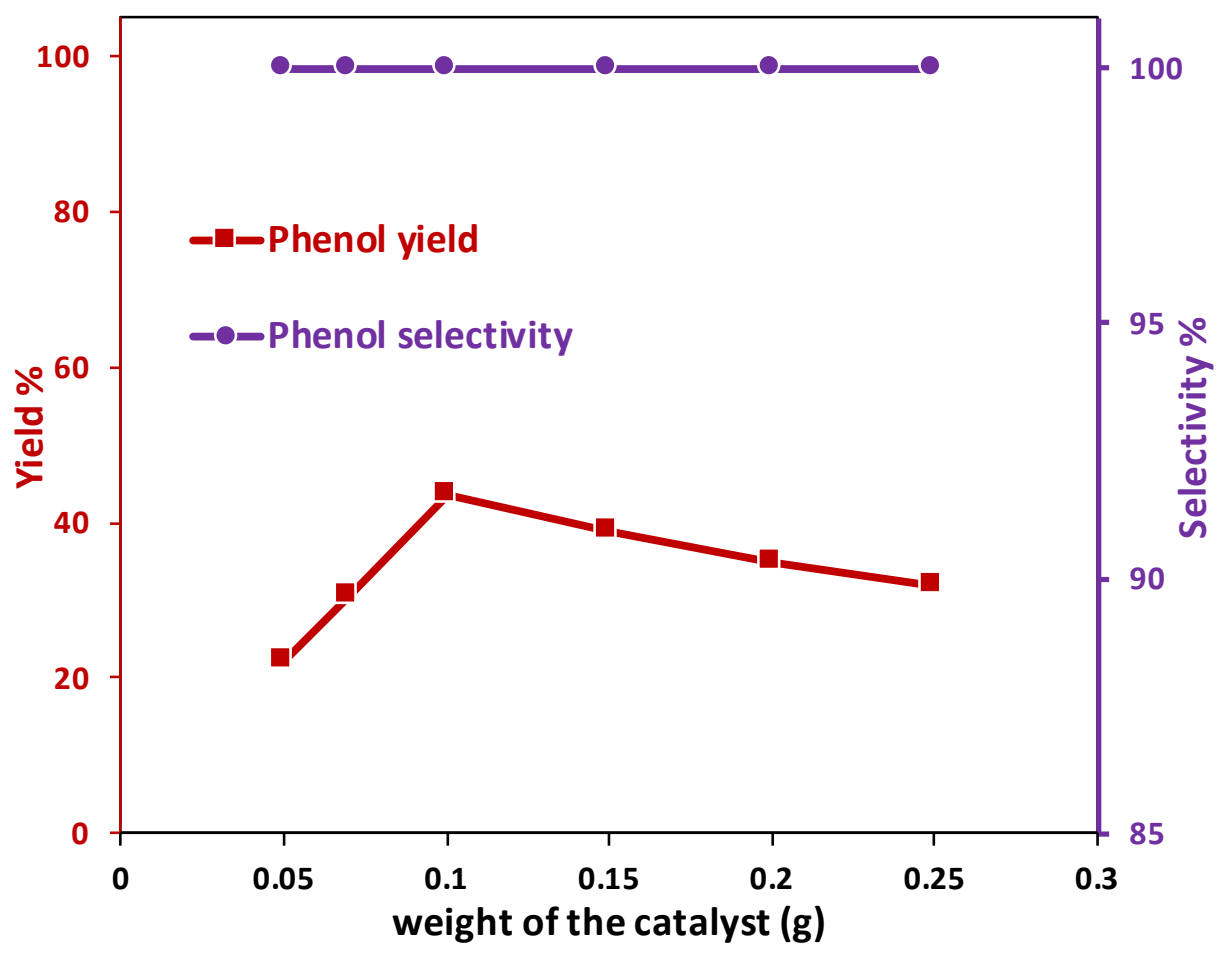

Figure 3. Impact of catalyst dosage. Reaction conditions: Benzene $(0.9 \mathrm{~mL})$, benzene: $\mathrm{H}_{2} \mathrm{O}_{2}$ $(1: 1)$, acetic acid $(1 \mathrm{~mL}), 60^{\circ} \mathrm{C}$ and $4 \mathrm{~h}$.

\section{Impact of benzene: $\mathrm{H}_{2} \mathrm{O}_{2}$ molar ratio}

To maximize the yield of phenol using $\mathrm{FeOCl}$ as a catalyst, we carefully optimized the benzene to hydrogen peroxide ratio as the addition of an excessive amount of hydrogen peroxide may have a negative impact on the oxidation process. Thus, it is highly recommended to carefully adjust the concentration of hydrogen peroxide to fulfil a maximum phenol yield. The impact of 
benzene: $\mathrm{H}_{2} \mathrm{O}_{2}$ molar ratio on the hydroxylation of phenol was examined in the range from 1:0.5 to $1: 2$ as displayed in Figure 4 . The obtained results reveal that the phenol yield is directly proportional to the benzene: $\mathrm{H}_{2} \mathrm{O}_{2}$ molar ratio, peaking at the molar ratio $1: 1$ and beyond this ratio the phenol yield declines.

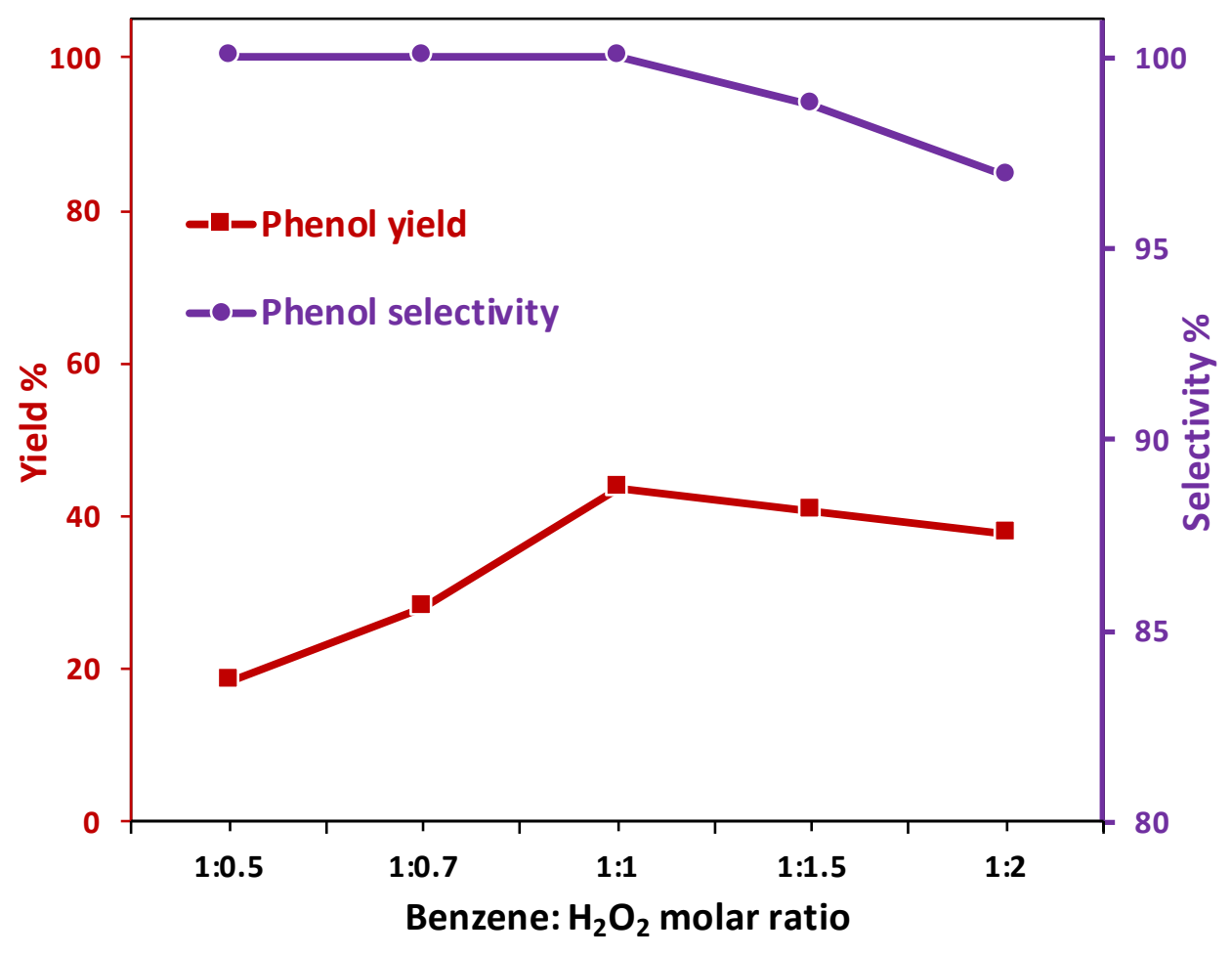

Figure 4. Impact of benzene: $\mathrm{H}_{2} \mathrm{O}_{2}$ molar ratio. Reaction conditions: Benzene $(0.9 \mathrm{~mL}), \mathrm{FeOCl}$ $(0.1 \mathrm{~g})$, acetic acid $(1 \mathrm{~mL}), 60^{\circ} \mathrm{C}$ and $4 \mathrm{~h}$.

It should be noted that the maximum $\mathrm{H}_{2} \mathrm{O}_{2}$ efficiency was detected at the molar ratio 1:1 in the presence of $\mathrm{FeOCl}$ as a catalyst as presented in Figure $\mathrm{S} 2$. The enhancement of the phenol yield upon increasing the benzene: $\mathrm{H}_{2} \mathrm{O}_{2}$ molar ratio is attributed to the presence of a considerable 
amount of $\mathrm{H}_{2} \mathrm{O}_{2}$ that produces higher amounts of hydroxyl radicals. However, the decline of the phenol yield upon the use of higher concentration of hydrogen peroxide is attributed to the presence of an overwhelming amount of $\mathrm{H}_{2} \mathrm{O}_{2}$ that scavenges the produced hydroxyl radicals with a rate, $\mathrm{k}=2.7 \times 10^{7} \mathrm{M}^{-1} \mathrm{~s}^{-1}$. The reaction of $\mathrm{H}_{2} \mathrm{O}_{2}$ with hydroxyl radicals gives rise to hydroperoxyl radicals. The generated hydroperoxyl radicals also reacts with hydroxyl radicals $\left(\mathrm{k}=6 \times 10^{9} \mathrm{M}^{-1} \mathrm{~s}^{-}\right.$ $\left.{ }^{1}\right)$ and consequently more hydroxyl radicals will be lost as presented in the following equations. ${ }^{40-43}$

$$
\begin{aligned}
& \mathrm{H}_{2} \mathrm{O}_{2}+\cdot \mathrm{OH} \rightarrow \mathrm{H}_{2} \mathrm{O}+\mathrm{HO}_{2}{ }^{\bullet} \\
& \mathrm{HO}_{2} \cdot+\cdot \mathrm{OH} \rightarrow \mathrm{H}_{2} \mathrm{O}+\mathrm{O}_{2}
\end{aligned}
$$

Another explanation or the decline of the phenol yield upon using an excessive concentration of $\mathrm{H}_{2} \mathrm{O}_{2}$ is the further of oxidation of the produced phenol to $p$-Benzoquinone. This explanation is supported by the decline of phenol selectivity upon increasing the benzene: $\mathrm{H}_{2} \mathrm{O}_{2}$ molar ratio. Thus, the produced radicals are involved in phenol oxidation instead of attacking the benzene molecules.

\section{Impact of reaction temperature}

The impact of reaction temperature on benzene hydroxylation was examined in the 30$80^{\circ} \mathrm{C}$ range to find out the lowest temperature that provides the maximum phenol yield as displayed in Figure 5. The adjustment of the reaction temperature in a catalytic system that contains hydrogen peroxide as an oxidant is a quite intricate operation. The use of elevated temperature leads to the decomposition of hydrogen peroxide into water and oxygen and consequently a great portion of the hydrogen peroxide will be lost. Screening the reaction temperature reveals that the phenol yield is directly proportional to the reaction reaching its climax at $60^{\circ} \mathrm{C}$, and beyond this temperature the phenol yield decreases. It can be argued that increasing the reaction temperature reduces the mass transfer limitation of hydroxyl radicals and thus the rate of the reaction between the benzene molecule and hydroxyl radicals is significantly enhanced. Furthermore, the oxidation rate of the 
organic compounds is greatly enhanced upon using elevated temperature ${ }^{44}$ However, the use of excessively elevated temperature such as $80^{\circ} \mathrm{C}$ has a negative impact on the phenol yield as the hydrogen peroxide is thermally decomposed into oxygen and water.

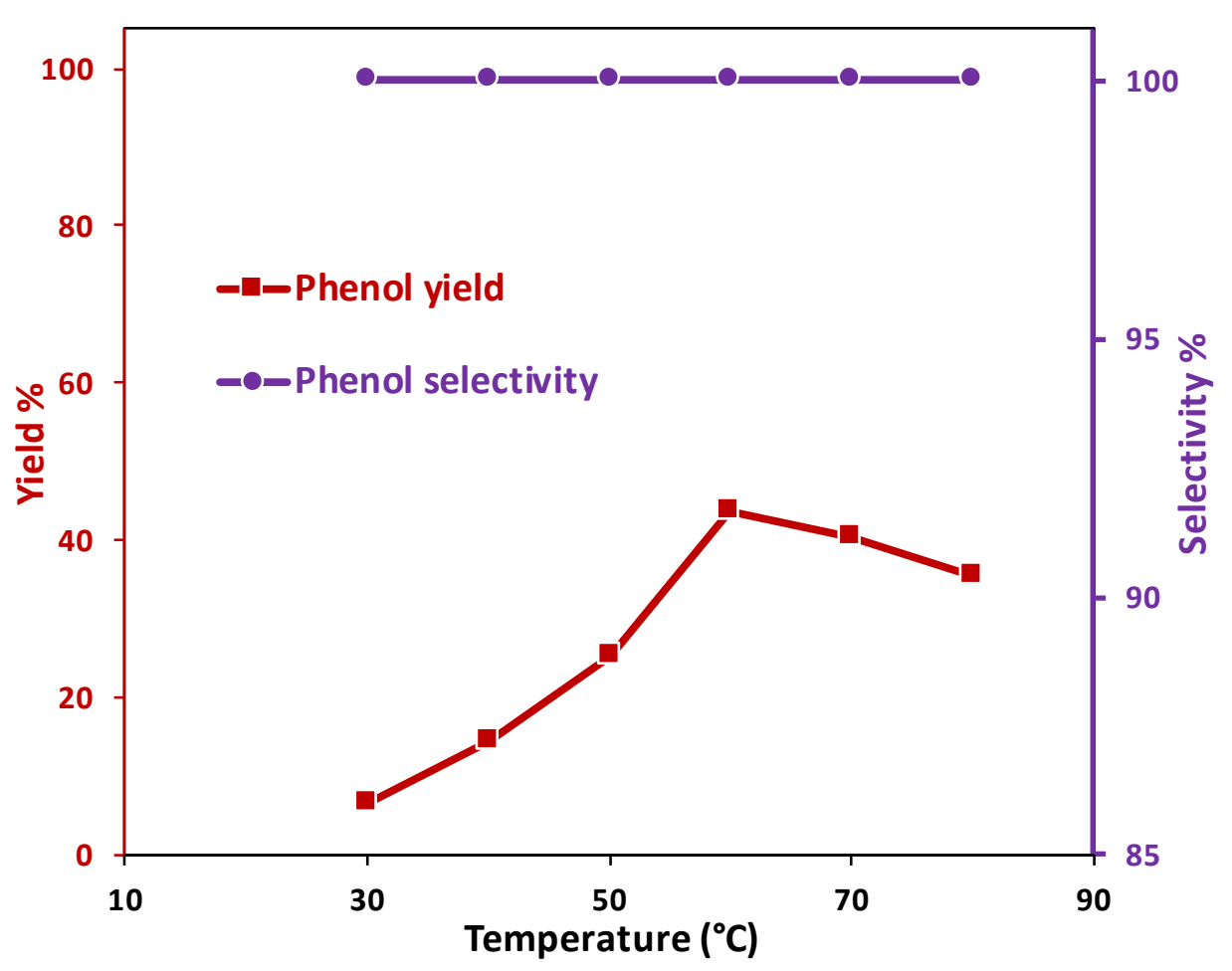

Figure 5. Impact of reaction temperature. Reaction conditions: Benzene (0.9 mL), FeOCl (0.1 g), benzene: $\mathrm{H}_{2} \mathrm{O}_{2}(1: 1)$, acetic acid $(1 \mathrm{~mL})$ and $4 \mathrm{~h}$.

\section{FeOCI recovery and reuse}

To check out the durability of the $\mathrm{FeOCl}$ in benzene hydroxylation, several experiments were carried out using the recovered $\mathrm{FeOCl}$ using the same employed conditions. FeOCl was recovered by centrifugation after each catalytic run, washed with ethanol and then dried at $120^{\circ} \mathrm{C}$ for $6 \mathrm{~h}$. 
The obtained results manifest that there is only a negligible decrease in the phenol yield after the fourth run using $\mathrm{FeOCl}$ catalyst under the same condition as displayed in Table S4. Thus, it can be inferred that the employed $\mathrm{FeOCl}$ is able to endure the harsh reaction condition of the benzene hydroxylation reaction. The phase of the recovered catalyst was investigated using XRD analysis to make sure that the catalyst did not suffer from any phase transformation during the catalytic activity and the obtained XRD patterns of the fresh and recovered catalyst is displayed in Figure S3. The obtained XRD pattern of the recovered catalyst reveals that did not undergo any phase transformation during benzene hydroxylation, however, a slight decrease in the peaks intensity was only observed.

\section{Insight into the mechanism of benzene hydroxylation using FeOCl}

As mentioned previously the efficacy of $\mathrm{FeOCl}$ in benzene hydroxylation is attributed to the unique ability of $\mathrm{FeOCl}$ for the production of a high amount of hydroxyl radicals in a short period of time and this process takes place via several successive steps. First, the facile self-redox potential of $\mathrm{FeOCl}$ fosters the prompt reduction of $\mathrm{Fe}^{3+}$ to $\mathrm{Fe}^{2+}$ upon the reaction with hydrogen peroxide (Scheme 1), which finally produces hydroperoxyl radical. ${ }^{45}$ The generated $\mathrm{Fe}^{2+}$ then reacts with hydrogen peroxide to produce hydroxyl radical and to eventually accomplish the redox cycle comprising the $\mathrm{Fe}^{2+} / \mathrm{Fe}^{3+}$ pair. It is to be noted that this redox cycle is deemed the essence of the homogeneous Fenton reaction where the reduction ferric ions to ferrous ions is the rate determining step that controls the reaction rate. ${ }^{46}$ 


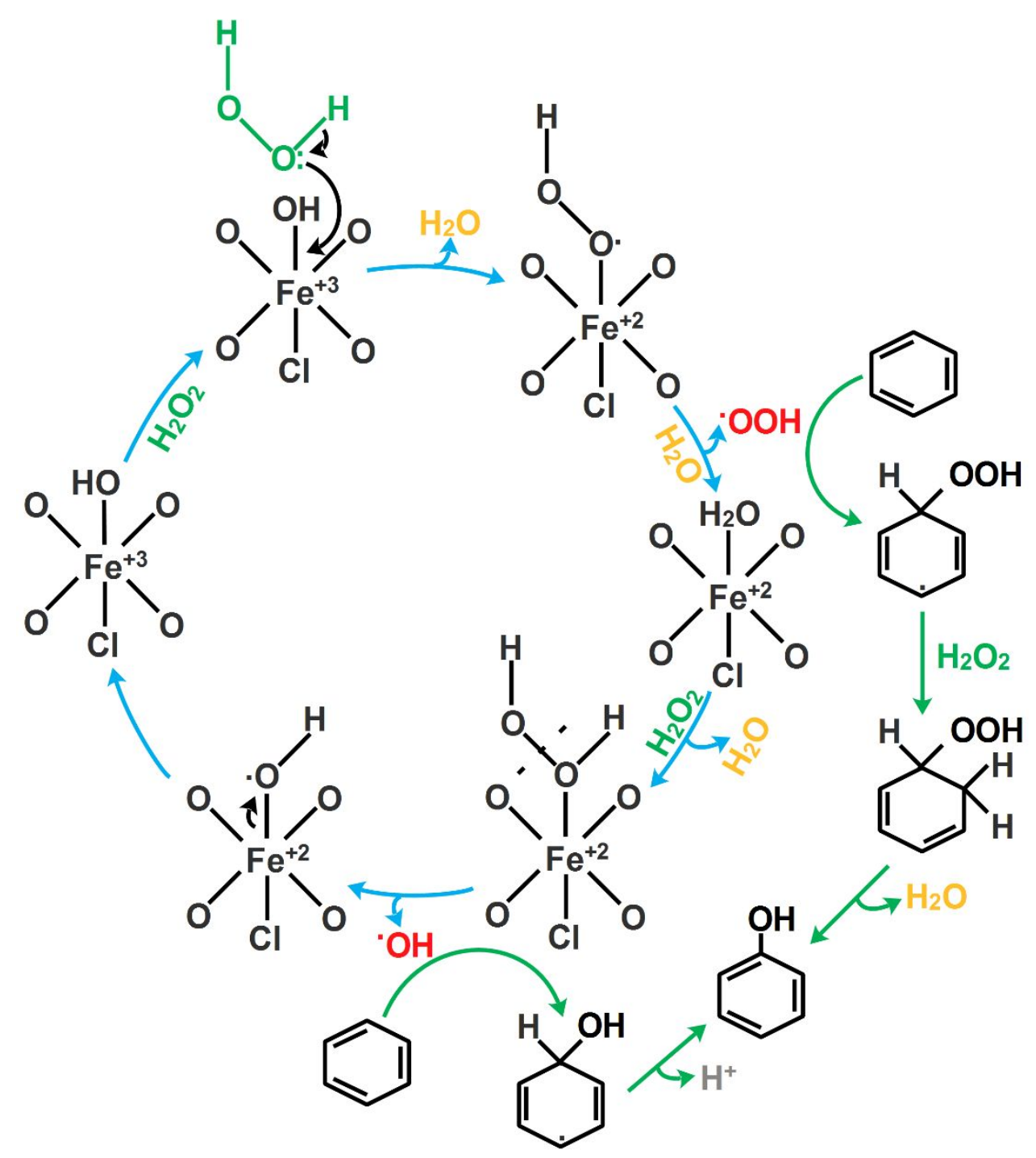

Scheme 1. Illustration of benzene hydroxylation mechanism using $\mathrm{FeOCl}$

Moreover, $\mathrm{FeOCl}$ enhances the production of hydroxyl radicals by dodging the undesirable oxidation of $\mathrm{Fe}^{2+}$ to $\mathrm{Fe}^{4+}$. It has been reported that $\mathrm{Fe}^{4+}$ could be formed as a result of the reaction of hydrogen peroxide and $\mathrm{Fe}^{2+}$ without producing hydroxyl radicals and thus this reaction was considered unfavorable. ${ }^{47}$ It should be mentioned that the existence of such reaction is reason that lies behind the inefficiency of a homogeneous Fenton catalyst at neutral $\mathrm{pH}$. Dodging such undesirable pathway directs the reaction between hydrogen peroxide and $\mathrm{Fe}^{2+}$ toward the production of hydroxyl radicals. The exposure of a huge number of unsaturated iron atoms over 
the plane (020) is considered the driving force for the prompt reduction of $\mathrm{Fe}^{3+}$ to $\mathrm{Fe}^{2+}$ and dodging the undesirable oxidation of $\mathrm{Fe}^{2+}$ to $\mathrm{Fe}^{4+}$ to produce hydroxyl radical. ${ }^{45}$ These exposed atoms can act as an active center for the activation of hydrogen peroxide. Moreover, the coordination of chlorine and oxygen boosts the reduction potential of the exposed iron atoms. Thus, electron transfer takes place more efficiently from the hydrogen peroxide molecule during the reduction of $\mathrm{Fe}^{3+}$ to $\mathrm{Fe}^{2+}$ and homolytic cleavage of hydrogen peroxide. ${ }^{48}$

In particular, the produced hydroxyl radical attack the benzene molecule to produce benzene-OH-adduct as presented in scheme 1. Next, benzene-OH-adduct produces phenol by losing hydrogen. On another hand, the generated hydroperoxyl radicals may also attack the benzene molecule to produce benzene-OOH-adduct. Thereafter, hydrogen peroxide reacts with the benzene-OOH-adduct that gives rise to a benzene-hydrogen-transferred OOH-adduct, which finally produces phenol by losing water molecule. ${ }^{49-50}$

\section{CONCLUSIONS}

In conclusion, selective hydroxylation of benzene to produce phenol with a high phenol yield (43.5\%) and high selectivity (100\%) has been fulfilled after only 4 h of hydroxylation using $\mathrm{FeOCl}$ in the presence of hydrogen peroxide as an oxidant. The exposure of a huge number of unsaturated iron atoms over the polar surface of $\mathrm{FeOCl}$ is considered the driving force for the prompt reduction of $\mathrm{Fe}^{3+}$ to $\mathrm{Fe}^{2+}$ and dodging the undesirable oxidation of $\mathrm{Fe}^{2+}$ to $\mathrm{Fe}^{4+}$ to produce considerable amount of hydroxyl radicals. The present work offers a felicitous strategy that can be an alternative for conventional processes concerned with the selective hydroxylation of benzene.

\section{ASSOCIATED CONTENT}


The Supporting Information is available free of charge on the ASC Publications website. Effects of reaction conditions on the selectivity and yield of phenol. Effects of reaction conditions on the selectivity of $p$-benzoquinone. Comparison between phenol selectivity and $p$-benzoquinone selectivity. Hydrogen peroxide efficiency. Comparison of catalytic activities with other catalysts for benzene hydroxylation. Reusability of $\mathrm{FeOCl}$ in benzene hydroxylation. XRD pattern of fresh and recovered catalyst.

\section{REFERENCES}

(1) Schmidt, R. J., Industrial catalytic processes-phenol production. Appl. Catal., A 2005, 280, 89-103.

(2) Yadav, G. D.; Asthana, N. S., Selective decomposition of cumene hydroperoxide into phenol and acetone by a novel cesium substituted heteropolyacid on clay. Appl. Catal., A 2003, 244, 341-357.

(3) Zhang, P.; Gong, Y.; Li, H.; Chen, Z.; Wang, Y., Selective oxidation of benzene to phenol by $\mathrm{FeCl}$ 3/mpg-C $3 \mathrm{~N} 4$ hybrids. RSC $A d v$. 2013, 3, 5121-5126.

(4) Pirutko, L.; Chernyavsky, V.; Uriarte, A.; Panov, G., Oxidation of benzene to phenol by nitrous oxide: Activity of iron in zeolite matrices of various composition. Appl. Catal., A 2002, 227, 143-157.

(5) Ye, X.; Cui, Y.; Wang, X., Ferrocene-Modified Carbon Nitride for Direct Oxidation of Benzene to Phenol with Visible Light. ChemSusChem 2014, 7, 738-742.

(6) Luo, G.; Lv, X.; Wang, X.; Yan, S.; Gao, X.; Xu, J.; Ma, H.; Jiao, Y.; Li, F.; Chen, J., Direct hydroxylation of benzene to phenol with molecular oxygen over vanadium oxide nanospheres and study of its mechanism. RSC Adv. 2015, 5, 94164-94170.

(7) Shul'pin, G. B.; Kozlov, Y. N.; Shul'pina, L. S.; Carvalho, W. A.; Mandelli, D., Oxidation reactions catalyzed by osmium compounds. Part 4 . Highly efficient oxidation of hydrocarbons and alcohols including glycerol by the $\mathrm{H} 2 \mathrm{O}$ 2/Os 3 (CO) 12/pyridine reagent. RSC Adv. 2013, 3, 15065-15074.

(8) Niwa, S.-i.; Eswaramoorthy, M.; Nair, J.; Raj, A.; Itoh, N.; Shoji, H.; Namba, T.; Mizukami, F., A one-step conversion of benzene to phenol with a palladium membrane. Science 2002, 295, 105-107.

(9) Balducci, L.; Bianchi, D.; Bortolo, R.; D'Aloisio, R.; Ricci, M.; Tassinari, R.; Ungarelli, R., Direct oxidation of benzene to phenol with hydrogen peroxide over a modified titanium silicalite. Angew. Chem. Int. Ed. 2003, 115, 5087-5090.

(10) Wen, G.; Wu, S.; Li, B.; Dai, C.; Su, D. S., Active sites and mechanisms for direct oxidation of benzene to phenol over carbon catalysts. Angew. Chem. Int. Ed. International Edition 2015, 54, 4105-4109.

(11) Yang, J.-H.; Sun, G.; Gao, Y.; Zhao, H.; Tang, P.; Tan, J.; Lu, A.-H.; Ma, D., Direct catalytic oxidation of benzene to phenol over metal-free graphene-based catalyst. Energ. Environ. Sci. 2013, 6, 793-798. 
(12) Jiaquan, X.; Huihui, L.; Ruiguang, Y.; Guiying, L.; Changwei, H., Hydroxylation of benzene by activated carbon catalyst. Chin. J. Catal. 2012, 33, 1622-1630.

(13) Chen, C.-h.; Xu, J.-q.; Jin, M.-m.; Li, G.-y.; Hu, C.-w., Direct synthesis of phenol from benzene on an activated carbon catalyst treated with nitric acid. Chin. J. Chem. Phys. 2011, 24, 358.

(14) Zhong, Y.; Li, G.; Zhu, L.; Yan, Y.; Wu, G.; Hu, C., Low temperature hydroxylation of benzene to phenol by hydrogen peroxide over Fe/activated carbon catalyst. J. Mol. Catal. A Chem. 2007, 272, 169-173.

(15) Zhong, Y.; Li, G.; Zhu, L.; Tang, D.; Hu, C., Direct catalytic hydroxylation of several typical aromatic compounds over Fe/activated carbon catalyst. Chem. J. Chinese U. 2007, 28, 1570.

(16) Jian, M.; Zhu, L.; Wang, J.; Zhang, J.; Li, G.; Hu, C., Sodium metavanadate catalyzed direct hydroxylation of benzene to phenol with hydrogen peroxide in acetonitrile medium. J. Mol. Catal. A Chem. 2006, 253, 1-7.

(17) Zhang, J.; Tang, Y.; Xie, J.-q.; Song, Z.-r.; Wang, L.; Hu, C.-w., The catalytic oxidation of phenol with $\mathrm{H} 2 \mathrm{O} 2$ by metalloporphyrins as peroxidase mimics. React. Kinet. Catal. L 2005, 85, 269-276.

(18) Zhang, J.; Tang, Y.; Xie, J.-Q.; Li, J.-Z.; Zeng, W.; Hu, C.-W., Study on phenol oxidation with H2O2. J. Serb. Chem. Soc. 2005, 70, 1137-1146.

(19) Zhang, J.; Tang, Y.; Li, G.; Hu, C., Room temperature direct oxidation of benzene to phenol using hydrogen peroxide in the presence of vanadium-substituted heteropolymolybdates. Appl. Catal., A 2005, 278, 251-261.

(20) Zhang, J.; Tang, Y.; Luo, Q.; Jian, M.; Hu, C., Study on the synthesis of heteropoly acids containing different amount of $\mathrm{V}$ and $\mathrm{Mo}$ and their catalytic performance for the direct hydroxylation of benzene to phenol. Chinese J. Inorg. Chem. 2004, 20, 935-940.

(21) Wang, C.; Hu, L.; Wang, M.; Yue, B.; He, H., Cerium promoted V-g-C3N4 as highly efficient heterogeneous catalysts for the direct benzene hydroxylation. R. Soc. open sci. 2018, 5 , 180371-180380.

(22) Hu, L.; Wang, C.; Yue, B.; Chen, X.; He, H., Vanadium-containing mesoporous carbon and mesoporous carbon nanoparticles as catalysts for benzene hydroxylation reaction. Mater. Today Commun. 2017, 11, 61-67.

(23) Wang, Y.; Zhang, T.; Li, B.; Jiang, S.; Sheng, L., Synthesis, characterization, electrochemical properties and catalytic reactivity of $\mathrm{N}$-heterocyclic carbene-containing diiron complexes. RSC Adv. 2015, 5, 29022-29031.

(24) Wu, L.; Zhong, W.; Xu, B.; Wei, Z.; Liu, X., Synthesis and characterization of copper (II) complexes with multidentate ligands as catalysts for the direct hydroxylation of benzene to phenol. Dalton Trans. 2015, 44, 8013-8020.

(25) Wen, N.; Xu, F.; Feng, Y.; Du, S., A new cumulene diiron complex related to the active site of Fe-only hydrogenases and its phosphine substituted derivatives: Synthesis, electrochemistry and structural characterization. J. Inorg. Biochem. 2011, 105, 1123-1130.

(26) Wang, D.; Wang, M.; Li, Z., Fe-based metal-organic frameworks for highly selective photocatalytic benzene hydroxylation to phenol. Acs Catal. 2015, 5, 6852-6857.

(27) Jourshabani, M.; Badiei, A.; Shariatinia, Z.; Lashgari, N.; Mohammadi Ziarani, G., Fesupported SBA-16 type cagelike mesoporous silica with enhanced catalytic activity for direct hydroxylation of benzene to phenol. Ind. Eng. Chem. Res. 2016, 55, 3900-3908. 
(28) Baykan, D.; Oztas, N. A., Synthesis of iron orthophosphate catalysts by solution and solution combustion methods for the hydroxylation of benzene to phenol. Mater. Res. Bull. 2015, 64, 294-300.

(29) Carneiro, L.; Silva, A. R., Selective direct hydroxylation of benzene to phenol with hydrogen peroxide by iron and vanadyl based homogeneous and heterogeneous catalysts. Catal. Sci. Technol. 2016, 6, 8166-8176.

(30) Al-Sabagh, A. M.; Yehia, F. Z.; Eshaq, G.; ElMetwally, A. E., Eclectic hydroxylation of benzene to phenol using ferrites of $\mathrm{Fe}$ and $\mathrm{Zn}$ as durable and magnetically retrievable catalysts. ACS Sustain. Chem. Eng. 2017, 5, 4811-4819.

(31) Herber, R.; Cassell, R., Synthesis, hyperfine interactions, and lattice dynamics of the intercalation compound (FeOCl (Kryptofix-21) 1/18. Inorg. Chem. 1982, 21, 3713-3716.

(32) Jarrige, I.; Cai, Y.; Shieh, S.; Ishii, H.; Hiraoka, N.; Karna, S.; Li, W.-H., Charge transfer in $\mathrm{FeOCl}$ intercalation compounds and its pressure dependence: An x-ray spectroscopic study. Phys. Rev. B 2010, 82, 165121.

(33) Hwang, S.; Li, W.-H.; Lee, K.; Lynn, J.; Wu, C.-G., Spiral magnetic structure of Fe in Van der Waals gapped $\mathrm{FeOCl}$ and polyaniline-intercalated FeOCl. Phys. Rev. B 2000, 62, 14157.

(34) Yang, X.-j.; Xu, X.-m.; Xu, J.; Han, Y.-f., Iron oxychloride (FeOCl): an efficient Fentonlike catalyst for producing hydroxyl radicals in degradation of organic contaminants. J. Am. Chem. Soc. 2013, 135, 16058-16061.

(35) Borah, P.; Datta, A.; Nguyen, K. T.; Zhao, Y., VOPO 4· 2H 2 O encapsulated in graphene oxide as a heterogeneous catalyst for selective hydroxylation of benzene to phenol. Green Chem. 2016, 18, 397-401.

(36) ElShafei, G. M. S.; Al-Sabagh, A. M.; Yehia, F. Z.; Philip, C. A.; Moussa, N. A.; Eshaq, G.; ElMetwally, A. E., Metal oxychlorides as robust heterogeneous Fenton catalysts for the sonophotocatalytic degradation of 2-nitrophenol. Appl. Catal. B Environ. 2018, 224, 681-691.

(37) ElMetwally, A. E.; Eshaq, G.; Al-Sabagh, A. M.; Yehia, F. Z.; Philip, C. A.; Moussa, N. A.; ElShafei, G. M. S., Insight into heterogeneous Fenton-sonophotocatalytic degradation of nitrobenzene using metal oxychlorides. Sep. Purif. Technol. 2019, 210, 452-462.

(38) Eggleston, C. M.; Stack, A. G.; Rosso, K. M.; Higgins, S. R.; Bice, A. M.; Boese, S. W.; Pribyl, R. D.; Nichols, J. J., The structure of hematite $(\alpha-\mathrm{Fe} 2 \mathrm{O} 3)(001)$ surfaces in aqueous media: scanning tunneling microscopy and resonant tunneling calculations of coexisting $\mathrm{O}$ and $\mathrm{Fe}$ terminations. Geochim. Cosmochim. Acta 2003, 67, 985-1000.

(39) Pignatello, J. J.; Oliveros, E.; MacKay, A., Advanced oxidation processes for organic contaminant destruction based on the Fenton reaction and related chemistry. Crit. Rev. Environ. Sci. Technol. 2006, 36, 1-84.

(40) Buxton, G. V.; Greenstock, C. L.; Helman, W. P.; Ross, A. B., Critical review of rate constants for reactions of hydrated electrons, hydrogen atoms and hydroxyl radicals $(\cdot \mathrm{OH} / \cdot \mathrm{O}-$ in aqueous solution. J. Phys. Chem. Ref. Data 1988, 17, 513-886.

(41) Mehrdad, A.; Hashemzadeh, R., Ultrasonic degradation of Rhodamine B in the presence of hydrogen peroxide and some metal oxide. Ultrason. Sonochem. 2010, 17, 168-172.

(42) Yehia, F.; Eshaq, G.; ElMetwally, A., Role of surface modification of some metal oxides with amino acids in upgrading the sonocatalytic degradation of nitrobenzene. Desalin. Water Treat. 2015, 56, 2160-2167.

(43) Yehia, F. Z.; Eshaq, G.; ElMetwally, A. E., Enhancement of the working pH range for degradation of p-nitrophenol using $\mathrm{Fe} 2+-$ aspartate and $\mathrm{Fe} 2+-$ glutamate complexes as modified Fenton reagents. Egypt. J. Pet. 2016, 25, 239-245. 
(44) Karimi, L.; Zohoori, S.; Yazdanshenas, M. E., Photocatalytic degradation of azo dyes in aqueous solutions under UV irradiation using nano-strontium titanate as the nanophotocatalyst. $J$. Saudi Chem. Soc. 2014, 18, 581-588.

(45) Sun, M.; Chu, C.; Geng, F.; Lu, X.; Qu, J.; Crittenden, J.; Elimelech, M.; Kim, J.-H., Reinventing Fenton Chemistry: Iron Oxychloride Nanosheet for pH-Insensitive H2O2 Activation. Environ. Sci. Technol. Lett. 2018, 5, 186-191.

(46) Kim, B. J.; Lee, D. U.; Wu, J.; Higgins, D.; Yu, A.; Chen, Z., Iron-and nitrogenfunctionalized graphene nanosheet and nanoshell composites as a highly active electrocatalyst for oxygen reduction reaction. J. Phys. Chem. C 2013, 117, 26501-26508.

(47) Koppenol, W.; Liebman, J. F., The oxidizing nature of the hydroxyl radical. A comparison with the ferryl ion (FeO2+). J. Phys. Chem. 1984, 88, 99-101.

(48) Li, H.; Shang, J.; Yang, Z.; Shen, W.; Ai, Z.; Zhang, L., Oxygen vacancy associated surface Fenton chemistry: Surface structure dependent hydroxyl radicals generation and substrate dependent reactivity. Environ. Sci. Technol. 2017, 51, 5685-5694.

(49) Hirose, K.; Ohkubo, K.; Fukuzumi, S., Catalytic Hydroxylation of Benzene to Phenol by Dioxygen with an NADH Analogue. Chem. Eur. J. 2016, 22, 12904-12909.

(50) Yamada, M.; Karlin, K. D.; Fukuzumi, S., One-step selective hydroxylation of benzene to phenol with hydrogen peroxide catalysed by copper complexes incorporated into mesoporous silica-alumina. Chem. Sci. 2016, 7, 2856-2863. 


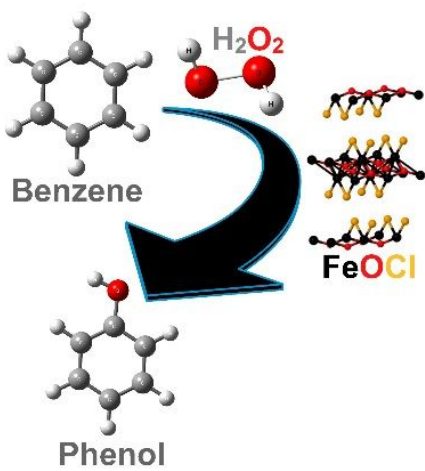

